\title{
Present Status of Exposure Tool Development for Low Energy Electron-beam Proximity Projection Lithography
}

\author{
Akihiro Endoa, Akira Higuchi", Haruo Kasaharaa", Hiroshi Nozuea, Nobuo Shimazua, \\ Toyoji Fukuib ${ }^{b}$, Naoki Yasumitsuc ${ }^{c}$, Tsutomu Miyatake ${ }^{c}$, and Norimichi Anazawa ${ }^{d}$ \\ $R \& D$ Division, ${ }^{a}$ LEEPL Corporation, \\ 3-1 Morinosato Wakamiya, Atsugi, Kanagawa 243-0124, Japan

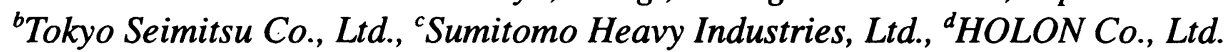

\begin{abstract}
A $\beta$-tool for Low Energy Electron-beam Proximity Projection Lithography (LEEPL) ${ }^{1-2}$ has been developed for proof of lithography (POL) of mass production tool, which is applied to required performance in 100-nm and 70-nm technology node. Major features of system design are an acceleration voltage of $1-5 \mathrm{kV}$, a beam current of 3-20 $\mu \mathrm{A}$, a maximum field size of $40 \mathrm{~mm}$ x $40 \mathrm{~mm}$ (effective field size of $25 \mathrm{~mm}$ x $25 \mathrm{~mm}$ ), a die-by-die alignment system and automatic loader systems for masks and wafers. The throughput is estimated over 20 wafers of $300 \mathrm{~mm}^{\phi}$ per hour. As examples of initial evaluation results, resolution of $45-\mathrm{nm} \mathrm{L/S}$ patterns and $48-\mathrm{nm}^{\phi}$ hole patterns in resist image were obtained. Alignment experiments are on going now, and overlay accuracy around $20-\mathrm{nm}(3 \sigma)$ over effective area of 8 -in. wafer is being expected.
\end{abstract}

Keyword: LEEPL, POL, die-by-die alignment system, throughput, resolution, overlay accuracy

\section{Introduction}

Several next generation lithography (NGL) technologies are proposed to achieve linewidths around $70-\mathrm{nm}$ and below. Though all of them have an ability to make such fine patterns just as required, they have the same issue that the lithography cost is drastically increasing because of the extreme rise of the production tool price and the running cost. LEEPL is only NGL candidate to meet the lithography requirements provided by the International Technology Roadmap for Semiconductors (ITRS), moreover to overcome this issue. This feature comes from the concepts described in section 2 .

One and half year ago, proof of concept (POC) of LEEPL was successfully completed by an alpha-tool which was developed in 4 months. Now a beta-tool for 100-nm and 70-nm technology node has been developed in a year and whose evaluation has been in progress. High-resolution and alignment capability has been already obtained. Moreover, 1X stencil masks around 70-nm dimensions are achieved by utilizing mask-making technology of the proximity $\mathrm{x}$-ray lithography (PXL). LEEPL has come close to practical use, so much earlier than other NGL candidates.

\section{Concepts and Advantages of LEEPL}

Figure 1 shows summary of concepts and advantages of LEEPL. There are three major system concepts of (a) use of a low energy e-beam. (b) use of a parallel e-beam and simple scan, (c) use of proximity projection.

Use of a low energy e-beam can drastically reduce the proximity effect caused by backscattered electron, which is usually the biggest problem for e-beam lithography. Because the proximity effect is negligible, LEEPL has no requirement for proximity effect correction. Besides, this concept can realize low power process, which reduce the heating expansion and damage of a mask and a resist film on wafers during exposure. In addition, it is easier to make 


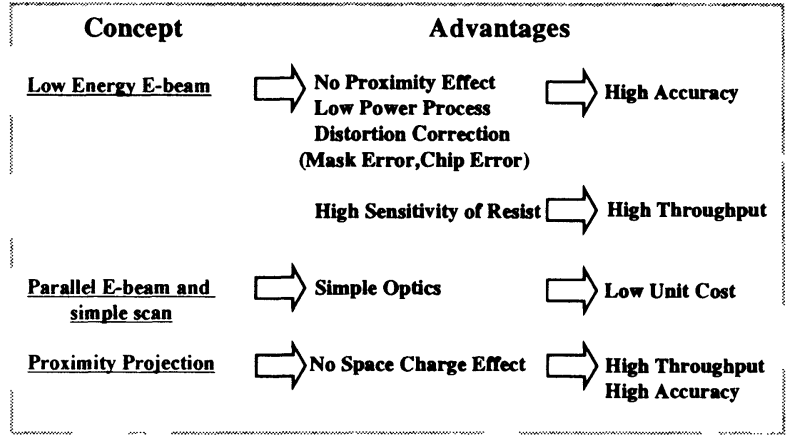

Figure 1. Summary of concepts and advantages.

mask patterns precisely because the mask membrane can be much thinner. Moreover, it is very easy to correct distortion of image placement on masks and wafer by changing the beam-landing angle under real-time control during exposure as described in section 4.1. These advantages promise to realize high accurate projection performance. On the other hand, the resist sensitivity can get much higher than that of any other e-beam lithography using higher acceleration voltage. So, this allows LEEPL to have high throughput capability.

Use of a parallel e-beam and simple scan can make electron optics and control system very simple. This advantage has capability to reduce the unit cost.

Finally, use of proximity projection can make space charge effect free at the path between a mask and a wafer. So, high beam current can be used for projection at this point. This advantage is another reason why LEEPL has high throughput capability.

\section{System Specifications}

The specifications of the $\beta$-tool, which is targeted for $100-\mathrm{nm}$ and $70-\mathrm{nm}$ technology generation, are shown in Table I. Major specifications of electron optics are an acceleration voltage of $1-5 \mathrm{kV}$ (fixed for usual use), a beam current of 3-20 $\mu \mathrm{A}$, a maximum field size of 40 $\mathrm{mm} \times 40 \mathrm{~mm}$ (effective field size of $25 \mathrm{~mm} \times 25$ $\mathrm{mm}$ for the $\beta$-tool). Specifications of projection accuracy are resolution of 50-nm, overlay accuracy of $25-\mathrm{nm}(|\mathrm{M}|+3 \sigma)$ by using a die-by-die alignment system. The throughput is estimated over 20 wafers of $300 \mathrm{~mm}^{\phi}$ per hour. Specifications of

$\begin{array}{ll}\text { 1. Target device DR } & 100-70 \mathrm{~nm} \\ \text { 2. Acceleration vol tage } & 1-5 \mathrm{kV} \\ \text { 3. Current for exposure } & 3 \sim 20 \mu \mathrm{A} \\ \text { 4. Maximum field size } & 40 \mathrm{~mm} \times 40 \mathrm{~mm} \\ & (\text { Effective area } 25 \mathrm{~mm} \times 25 \mathrm{~mm}) \\ \text { 5. Resolution } & 50 \mathrm{~nm} \\ \text { 6. Overlay accuracy } & 25 \mathrm{~nm} \text { (IMeanl }+3 \sigma) \\ & \text { (Die-by-die alignment) } \\ \text { 7. Throughput } & \text { over } 20 / \mathrm{hr} @ 300 \mathrm{~mm} \text { wafer } \\ \text { (Production Tool } & \text { over } 40 / \mathrm{hr} @ 300 \mathrm{~mm} \text { wafer) } \\ \text { 8. Mask size } & 4 \text { inch and } 8 \text { inch } \\ \text { 9. Wafer size } & 200 \text { or } 300 \mathrm{~mm} \text { wafer }\end{array}$

Table I. Major specifications of a $\beta$-tool.

substrates are 4-in. and 8-in. size of masks, 200 $\mathrm{mm}^{\phi}$ or $300 \mathrm{~mm}^{\phi}$ size of wafers, which are handled by automatic loader systems.

\section{System Configurations and Design}

As shown in figure 2 , the $\beta$-tool consists of five major modules, which are electron optical column, alignment system with peripheral instruments, $X-Y$ stage, automatic mask and wafer loader, and total control system.

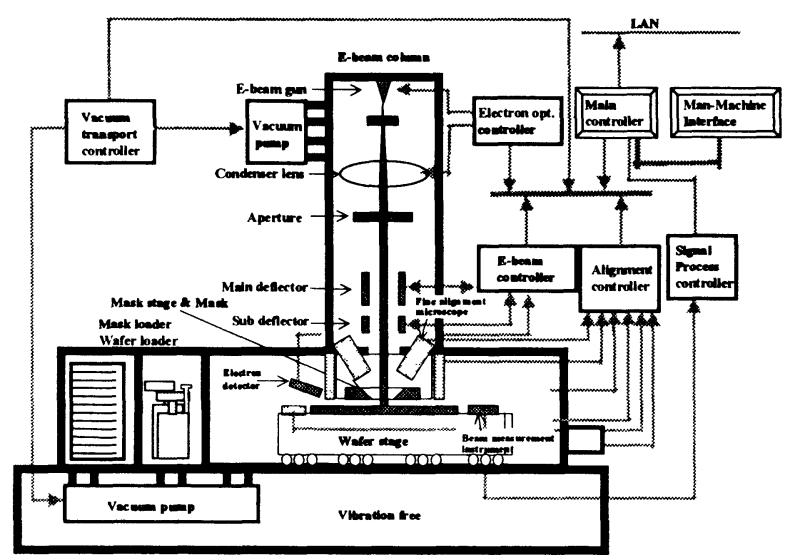

Figure 2. Block diagram of a $\beta$-tool.

\subsection{Electron Optical Column and Control System}

As shown in figure 3 , the electron column consists of an e-beam gun with $\mathrm{LaB}_{6}$ cathode, a condenser lens, two position of variable beam limiting aperture, and two sets of electrostaticoctapole deflector, which are main deflectors and sub-deflectors. The concepts of LEEPL described above can make the column very simple, just as shown in figure 4 . 


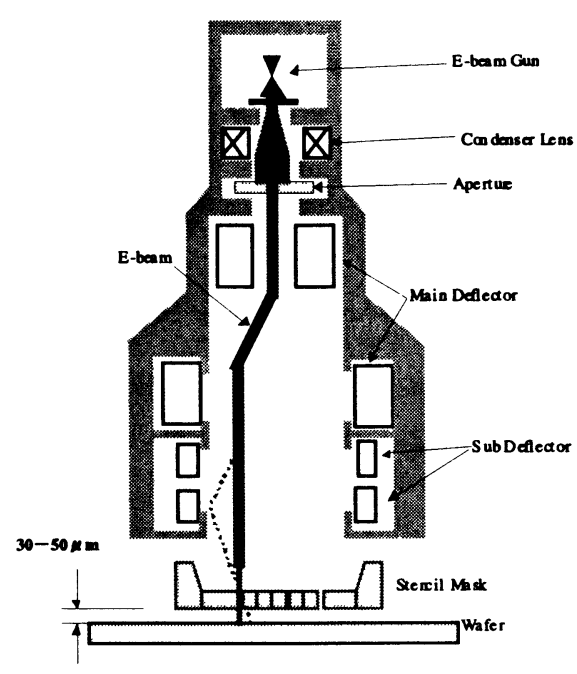

Figure 3. Schematic illustration of an electron optical column.

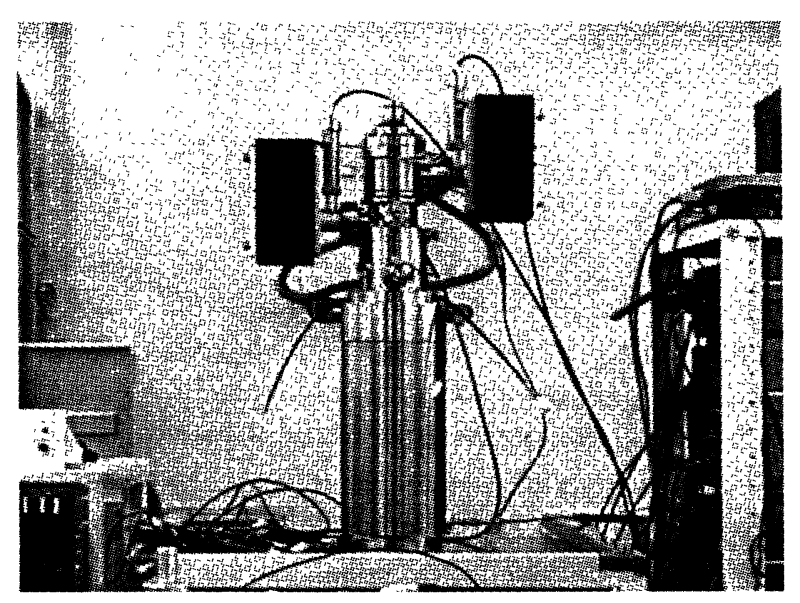

Figure 4. Photograph of the column.

The e-beam source of $\mathrm{LaB}_{6}$ is good enough for getting high beam current with satisfactory stability. Figure 5 shows the evaluation result of the beam current stability at $23.2 \mu \mathrm{A}$. The current variations of every one-hour period were less than $0.5 \%$ during 12 hours. Figure 6 shows a beam shape measured by faraday cage with pinhole mask on it. This beam shape was like columnar shape and the beam diameter was $450 \mu \mathrm{m}^{\phi}$ of FWHM.

Main deflectors are used for scanning a beam over a mask and a wafer with a stage motion by step and repeat. Sub-deflectors are used for tilting a beam as illustrated in figure 3 in order to correct distortion of pattern placement on both a mask and a wafer. Figure 7 shows an example of magnification correction in case of reduction by this way. Sub-deflectors on the $\beta$-tool have capability to control the beam-landing angle with range of $\pm 10 \mathrm{mrad}$. Moreover, this function is very effective to correct the mechanical alignment error caused on alignment system described in section 4.2 to achieve high accurate overlay.

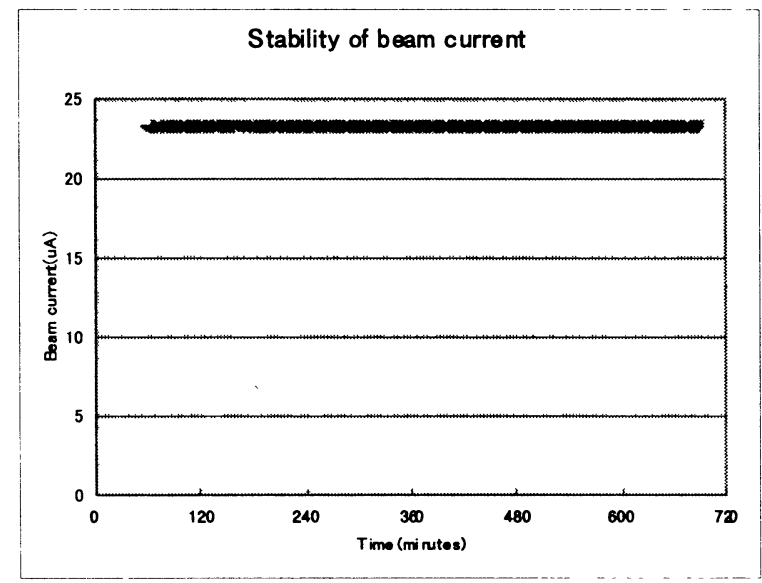

Figure 5. Stability of beam current.

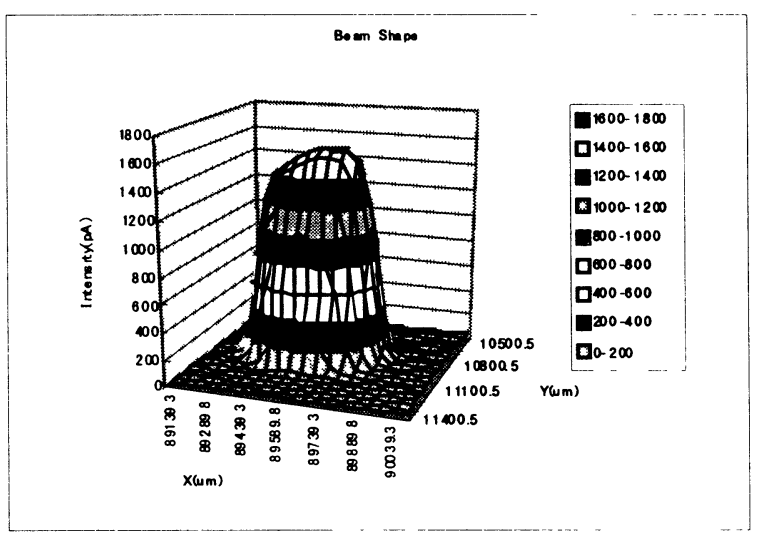

Figure 6. Beam shape.

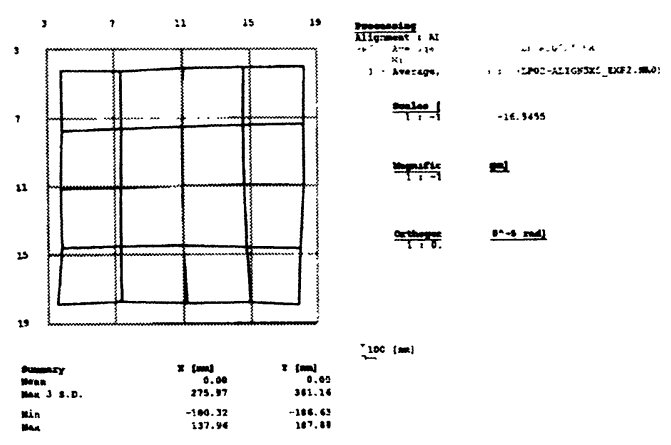

Figure 7. An example of magnification correction

\subsection{Alignment System}

A video-based scattered-light alignment (SLA) system ${ }^{3-4}$, which has been used on PXL stepper and experienced in actual device processes, is used for die-by-die alignment on the $\beta$-tool. This 
system consists of major sub-modules of a mask stage and a wafer stage for fine motion, alignment microscopes, a signal processing system and peripheral instruments such as coarse alignment microscopes and height detectors for mask and wafer.

Figure 8 shows an alignment microscope, which has a size of $300 \mathrm{~mm}(\mathrm{~L}) \times 70 \mathrm{~mm}(\mathrm{~W}) \times 90 \mathrm{~mm}$ (H). Four alignment microscopes are mounted outside of the exposure field with tilted angle for $\mathrm{X}-\mathrm{Y}-\theta-\mathrm{Mag}$. automatic alignment as shown in figure 9-(a). An incoherent white light illumination (400-800 $\mathrm{nm}$ wavelength) is used for the alignment. Incident light is scattered at the mark edges on a mask and a wafer and the scattered lights from both the mask and the wafer are collected by the objective of the alignment microscope simultaneously as shown in figure 9(b). A CCD observes this image formed by the objective as shown in figure 9-(c).

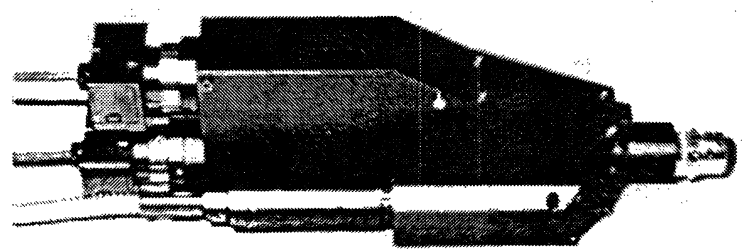

Figure 8. Photograph of alignment microscope.

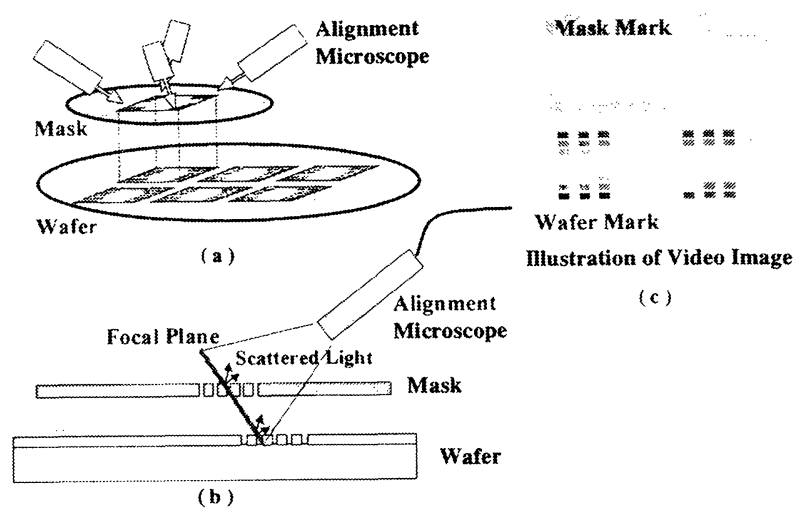

Figure 9. (a) Arrangement of alignment microscopes, (b) Schematic of alignment method and (c) Illustration of alignment video images.

The mechanical alignment is proceeded as the mask mark signal is aligned to center of the pair of wafer mark signals by controlling fine motions of a mask stage and a wafer stage with piezo drives as shown in figure 10. The relative position of the signals of the mask mark and wafer mark is monitored and fed-back to both the mask stage and the wafer stage to maintain the mechanical

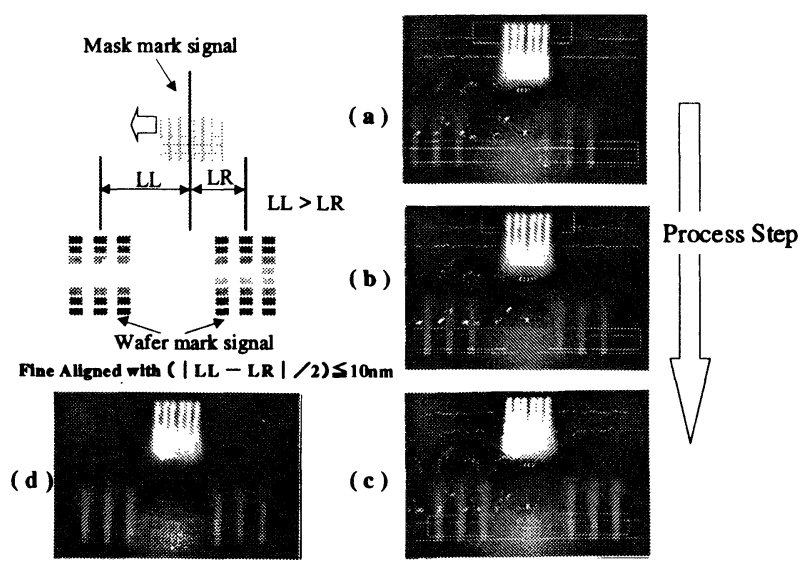

Figure 10. Alignment process.

alignment error less than 10-nm during full time of exposure. The residual error is corrected by subdeflectors to tilt the beam-landing angle with a feedback loop between alignment system and deflection control system.

Major advantages of this alignment system are (a) capability to monitor mechanical alignment status directly during exposure with feedback loop to align, (b) capability to apply position sensing to various gaps between a mask and a wafer and to monitor the gap simultaneously, (c) insensitivity to multiple reflection and diffracted light between a mask and a wafer by using white light illumination. These advantages lead to high accurate alignment performance with robustness.

\subsection{X-Y Stage}

$\mathrm{X}$-Y wafer stage for coarse motion employs nonmagnetic ultrasonic linear motors, on which there is the fine stage of the alignment system described above. The whole stage system can be taken out from main chamber for maintenance because of no motor connections through the chamber wall. The $\mathrm{X}$ and $\mathrm{Y}$ tables are made of a ceramic to achieve accurate addressing of beam scan. The maximum stage velocity and the acceleration are $100 \mathrm{~mm} / \mathrm{s}$ and $0.1 \mathrm{G}$. Both eight-inch and twelveinch wafers can be loaded by changing pallets without neither any exchange of parts nor any mechanical adjustments.

\subsection{Mask and Wafer Loader}

A mask loader system consists of a SMIF pod opener as shown in figure 11 , a pre-aligner, and a mask-handling robot in a vacuum load chamber. Four-inch and eight-inch size of masks can be loaded to the system. A SMIF pod is used as a 
mask case to keep a mask particle free during handling outside of the system. In this system, slow pumping and venting functions are used for taking care of particle adhesion on a mask during loading and unloading.

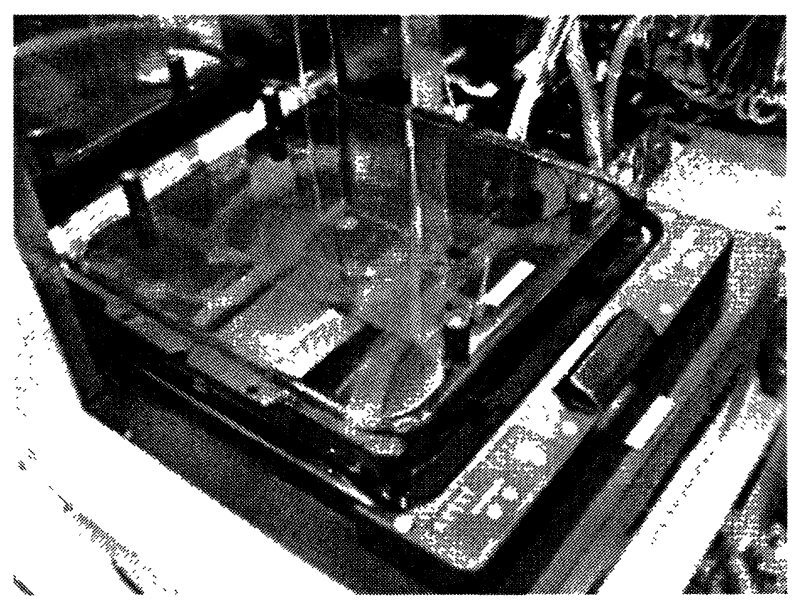

Figure 11. SMIF pod opener of mask loader.

A wafer loader system consists of a minienvironment unit as shown in figure 12 , a pre-aligner, and a pallet exchanger in a vacuum load chamber. The mini-environment unit has two open cassettes for eight-inch wafers or two FOUPs for twelve-inch wafers and has chemical filter for chemical amplified resist. The pallet exchanger has two ports for pallet loading and unloading to/from the wafer stage to minimize the overhead time. The slow pumping and venting functions are also employed on the vacuum load chamber of the pallet exchanger.

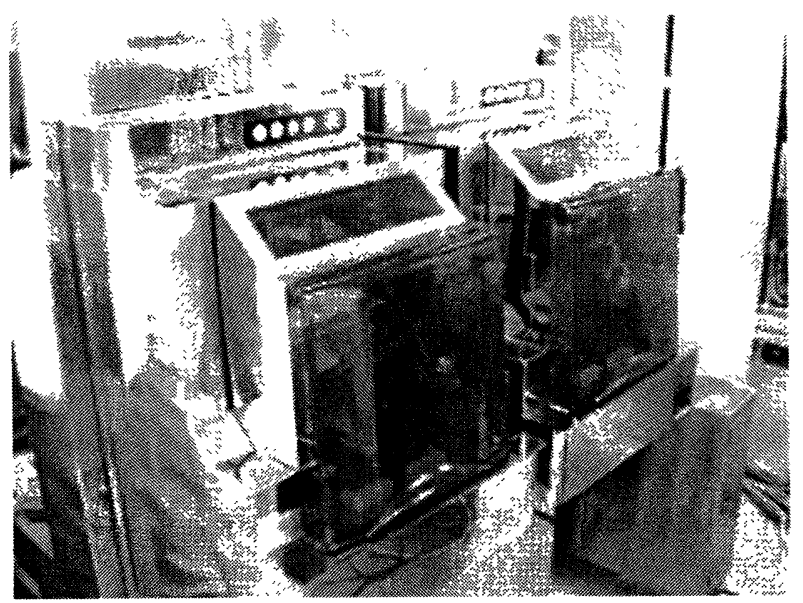

Figure 12. Mini-environment unit of wafer loader.

\subsection{Total Control System}

A total control system consists of a main controller and a man-machine interface. The electron optics controller, the e-beam controller, the vacuum transport controller, alignment controller, and signal process controller are obeyed the main controller as shown in figure 2 . The major functions of main controller are change of video signals concerned with die-by-die alignment and e-beam adjustments, vacuum control over main chamber and mask/wafer load chamber, calibration of e-beam and deflection characteristics, sub-system control including initializing, mask and wafer loading/unloading, and exposure execution. The man-machine interface is used for operations of adjustments of each sub-system, registrations of alignment models, creations and edits of recipe file of exposure, monitoring the sub-systems status, and controlling data files and parameter files.

\section{Exposure Examples}

\subsection{Proximity Effect}

The proximity effect was evaluated by using bilayer resist process as actual device fabrication process for LEEPL. The top imaging layer was silicon-containing negative tone resist of $130-\mathrm{nm}$ thickness and the bottom layer was novolac type of photo-resist of 350-nm thickness. There was not seen proximity effect as shown in figure 13. The $180-\mathrm{nm}$ isolated line was fabricated with independent of patterns around it.

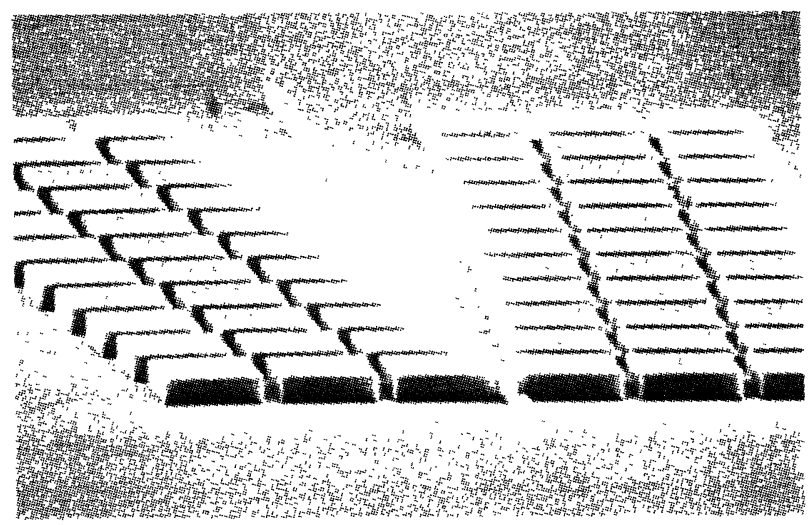

Figure 13. Proximity effect evaluation.

\subsection{Resolution and line edge roughness}

Figure 14 shows the resolution performance evaluated by line and space patterns in resist image. The 45-nm line and 115-nm space patterns and 70$\mathrm{nm}$ line and space patterns (1:1 replication), which were almost as same size as those on a mask, were obtained. Figure 15 shows the resolution performance evaluated by hole patterns in resist image. Hole patterns of $48-\mathrm{nm}^{\phi}$ size or below 
were obtained.

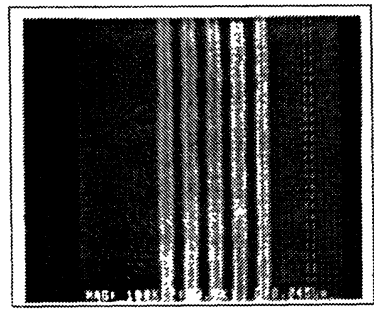

(a)

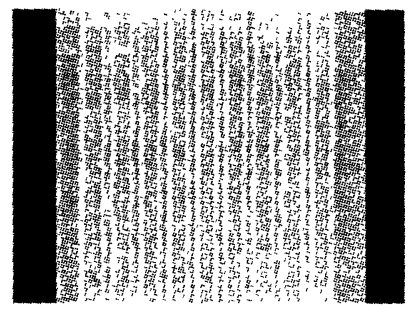

(b)
Figure 14. Resolution evaluations by (a) $45-\mathrm{nm} \mathrm{L} / \mathrm{S}$ (160-nm pitch) and (b) 70-nm L/S (140-nm pitch)

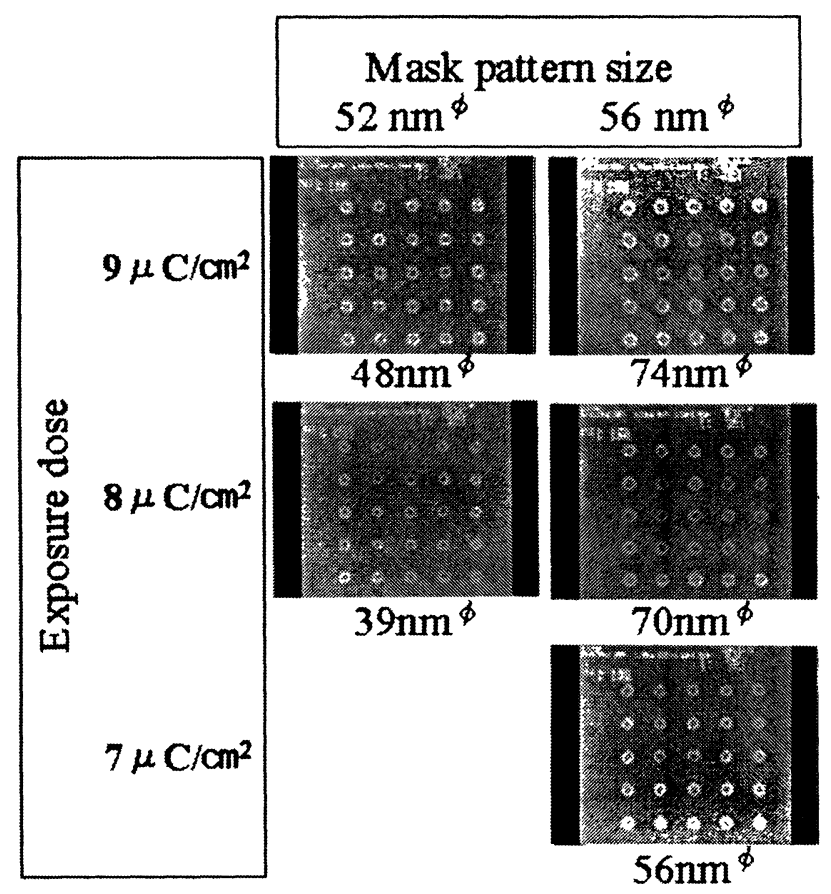

Figure 15. Resolution evaluations by hole patterns

The line and space patterns and hole patterns were fabricated by 50 -nm thickness resist film of ZEP520 (Nippon Zeon Co., Ltd.).

Line edge roughness (LER) was improved drastically for about a year as shown in figure 16 .

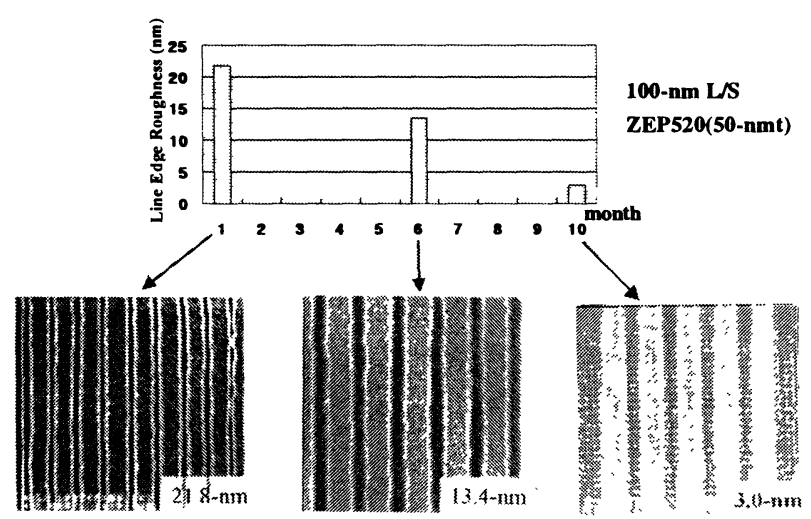

Figure 16. Line edge roughness evaluations.
The resist process was quite same as that of resolution evaluation. The 3-nm of LER was obtained by the $\beta$-tool at 10 month later from initial exposure on $\alpha$-tool. The others were obtained by $\alpha$-tool. Improvements of both characteristic of e-beam incident angle distribution and mask pattern quality are thought as major cause for this LER improvement.

The resolution capability and LER quality were proved as satisfied for 70-nm technology node.

\subsection{Overlay}

Figure 17 shows example of mechanical alignment error in $\mathrm{x}$-direction generated when a mask and a wafer are interlocked by alignment system and the closed-feedback loop is executed. The alignment error values were almost less than 15-nm and expected to be improved less than 10$\mathrm{nm}$ by adjustments of alignment system. The residual error of this is to be corrected by subdeflectors.

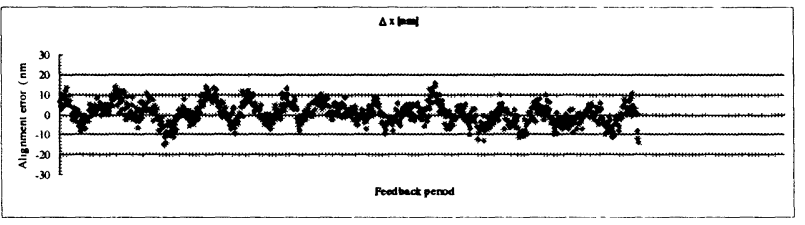

Figure 17. Mechanical alignment error during closedfeedback execution

Alignment experiments are on going now, and overlay accuracy around $20-\mathrm{nm}(3 \sigma)$ over effective area of 8-in. wafer is being expected.

\section{Conclusion}

A $\beta$-tool for Low Energy Electron-beam Proximity Projection Lithography (LEEPL) has been developed for proof of lithography (POL) of mass production tool. Major system functions and operations were confirmed by exposure examples. As examples of initial evaluation results, resolution of $45-\mathrm{nm} \mathrm{L} / \mathrm{S}$ patterns and 48$\mathrm{nm}^{\phi}$ hole patterns in resist image were obtained. Alignment experiments are on going now, and overlay accuracy around $20-\mathrm{nm}(3 \sigma)$ over effective area of 8 -in. wafer is being expected. This needs further investigations. 


\section{Acknowledgments}

The authors wish to thank Dr. M. Oda, and Mr. S. Takahashi of NTT advanced Technology Corporation for their development of LEEPL masks and their support in the evaluations of patterning resolution. The authors are also grateful to co-workers in Tokyo Seimitsu Co., Ltd., Sumitomo Heavy Industries, Ltd., and HOLON Co., Ltd.

\section{References}

1. T.Utsumi, Jpn. J. Appl. Phys. Vol.38 7046 Part 1, No. 12B, December 1999.

2. T.Utsumi, J.Vac. Sci. Technol. B 17(6), pp.28972902, Nov/Dec 1999.

3. T.Miyatake et al, SPIE Proceedings Vol. 3048, pp.225-236, March 1997.

4. T.Miyatake et al, J.Vac. Sci. Technol. B 15(6), pp.2471-2475, Nov/Dec 1997. 